\title{
Higher-Order Voronoi Diagrams on Triangulated Surfaces
}

\author{
Sergio Cabello* $\quad$ Marta Fort ${ }^{\dagger} \quad$ J. Antoni Sellarès ${ }^{\ddagger}$
}

January 12, 2009

\begin{abstract}
We study the complexity of higher-order Voronoi diagrams on triangulated surfaces under the geodesic distance, when the sites may be polygonal domains of constant complexity. More precisely, we show that on a surface defined by $n$ triangles the sum of the combinatorial complexities of the order- $j$ Voronoi diagrams of $m$ sites, for $j=1, \ldots, k$, is $O\left(k^{2} n^{2}+k^{2} m+k n m\right)$, which is asymptotically tight in the worst case.
\end{abstract}

\section{Introduction}

The diverse generalizations of Voronoi diagrams have important applications in several fields and application areas, such as computer graphics, geometric modelling, geographic information systems, visualization of medical data-sets, pattern recognition, robotics and shape analysis. Given a set of $m$ primitives, called sites, in an ambient space equipped with a metric, the order- $k$ Voronoi diagram, where $k$ is an integer between 1 and $m-1$, partitions the ambient space into regions such that each point within a fixed region has the same set of $k$ closest sites. Many variants of these diagrams can be found in the literature by considering various ambient spaces, taking sites of different shape or nature, associating weights to the sites, or changing the underlying metrics $[3,4,11]$. In this paper we concentrate on Voronoi diagrams defined on triangulated surfaces for polygonal sites of constant-description complexity.

Surfaces, sites, and bisectors. Let $\mathcal{P}$ be a triangulated polyhedral surface consisting of $n$ triangles. The surface $\mathcal{P}$ may have nonzero genus. The distance $d(p, q)$ between two points $p, q \in \mathcal{P}$ of the surface is given by their geodesic distance, that is, the length of the shortest path between the two points that is contained in the surface.

In this paper, a site $s$ in $\mathcal{P}$ is a polygonal domain contained in $\mathcal{P}$ with constant-description complexity; it may be a point, a segment, a polygonal path with a bounded number of segments, or a domain enclosed by a closed polygonal path with a bounded number of segments.

The distance $d(p, s)$ from a point $p \in \mathcal{P}$ to a site $s$ is defined by the length of the shortest path from $p$ to any point of $s$, that is, $d(p, s)=\min _{q \in s} d(p, q)$. The bisector $\beta\left(s, s^{\prime}\right)$ defined by sites $s$ and $s^{\prime}$ is the locus of points at equal distance from $s, s^{\prime}$.

\footnotetext{
*Department of Mathematics, IMFM and FMF, University of Ljubljana, Slovenia, sergio.cabello@fmf.uni-lj.si; partially supported by the Slovenian Research Agency, project J1-7218 and program P1-0297.

${ }^{\dagger}$ Institut d'Informàtica i Aplicacions, Universitat de Girona, Spain, mfort@ima.udg.edu.

${ }^{\ddagger}$ Institut d'Informàtica i Aplicacions, Universitat de Girona, Spain, sellares@ima.udg.edu; supported by the Spanish MEC grant TIN2007-67982-C02-02.
} 
A terrain $\mathcal{T}$ is a polyhedral surface embedded in $\mathbb{R}^{3}$ that is homeomorphic to a topological disk and is $x y$-monotone - a vertical line intersects $\mathcal{T}$ at most once. The domain of a terrain is the projection of $\mathcal{T}$ onto the $x y$-plane.

Let $S=\left\{s_{1}, \ldots, s_{m}\right\}$ be a set of $m$ sites in $\mathcal{P}$. We make the following assumptions:

- The sites are pairwise disjoint.

- No vertex of the surface is equidistant from two sites.

- No point of the surface is equidistant from four sites.

When any of the first two assumptions is removed, it may happen that a bisector defined by two sites contains two-dimensional pieces. With the first two assumptions, a bisector does not contain two-dimensional pieces and consists of straight-line, parabolic and hyperbolic arcs $[6,8]$. A breakpoint of a bisector is an intersection point between two adjacent arcs of the bisector. A breakpoint corresponds either to a point where the bisector crosses an edge of $\mathcal{P}$ or to a point where there are two different shortest paths from one of the sites defining the bisector ${ }^{1}$. According to previous results $[6,8]$, a bisector has $O\left(n^{2}\right)$ breakpoints.

Order- $k$ Voronoi diagram. We are interested in the order- $k$ Voronoi diagrams of the sites $S$. We next recall its definition, together with other related concepts. For a subset $S^{\prime}$ of the sites, the Voronoi region $V\left(S^{\prime}\right)$ of $S^{\prime}$ is defined as

$$
\left\{p \in \mathcal{P} \mid d\left(p, s^{\prime}\right) \leq d(p, s), \forall s^{\prime} \in S^{\prime}, \forall s \in S \backslash S^{\prime}\right\} .
$$

For each integer $1 \leq k \leq m-1$, the order- $k$ Voronoi diagram of $S, \operatorname{Vor}_{k}(S)$, is the family of nonempty Voronoi regions $V\left(S^{\prime}\right)$, where $S^{\prime}$ ranges over all subsets of $S$ with $k$ sites. When $k=1$ and $k=m-1$ the order- $k$ Voronoi diagrams are called the (closest-site) Voronoi and the furthestsite Voronoi diagram, respectively. A $k$-region is a Voronoi region in the order- $k$ Voronoi diagram. A $k$-face is a maximal connected component of a $k$-region. A $k$-edge is a connected component in the intersection of two $k$-faces. A $k$-edge always lies on the bisector of two sites. A $k$-breakpoint is a breakpoint on a $k$-edge. A $k$-vertex is the intersection point of three or more $k$-faces.

The terms faces, edges, and vertices of the order- $k$ Voronoi diagram are chosen because of their resemblance with a graph embedded in the surface $\mathcal{P}$. The removal of all $k$-edges from the surface leaves a collection of connected sets. The closure of one such connected set is a $k$-face, and vice versa. Recall the third assumption we have made above: no point of the surface is equidistant from four sites. In this case, no four bisectors intersect at a common point, and hence a $k$-vertex has precisely three $k$-edges arriving to it. Finally, let us remark the difference between vertices and breakpoints: while vertices correspond to endpoint of edges, breakpoints can only appear along the relative interior of edges.

The (combinatorial) complexity of the order- $k$ Voronoi diagram is the total number of $k$-faces, $k$-edges, $k$-vertices, and $k$-breakpoints.

Our results. Let $\operatorname{Vor}_{\leq k}(S)$ denote the family of all order- $j$ Voronoi diagrams of $S$ for $j=1, \ldots, k$. Let us define the complexity of $\operatorname{Vor}_{\leq k}(S)$ as the sum of the complexities of the order- $j$ Voronoi diagrams, $j=1, \ldots, k$. We show that the complexity of $\operatorname{Vor}_{\leq k}(S)$ is $O\left(k^{2} n^{2}+k^{2} m+k n m\right)$, and that this bound is asymptotically tight in the worst case. We also prove that the complexity of $\operatorname{Vor}_{\leq k}(S)$ in so-called realistic terrains (the concept is explained in Section 4) is $O\left(k^{2} n+k \sqrt{n} m+k^{2} m\right.$ ).

\footnotetext{
${ }^{1}$ This definition of breakpoint is consistent with [1] but different from [2, 9].
} 
Related work. In the Euclidean plane $\mathbb{R}^{2}$, which can be regarded as a surface of constant complexity, the order- $j$ Voronoi diagram of a set of $m$ points has complexity $O(j(m-j))$, and hence the complexity of $\operatorname{Vor}_{\leq k}(S)$ is $O\left(m k^{2}\right)$ [7, 12]. In the case of a triangulated surface consisting of $n$ triangles and a set of $m$ point sites, the complexity of the closest-site Voronoi diagram is $O(n(n+m))$, while the number of vertices is $O(m)[8,10]$. For surfaces of genus zero, Aronov et al. [1] show that the furthest-site Voronoi diagram has complexity $\Theta\left(m n^{2}\right)$ in the worst-case. These previous results for surfaces were written for the case where the sites are points; in this case the edges consist of straight-line and hyperbolic arcs. Combining approaches in $[6]$ and $[1,8]$, the results directly extend to segment, polygonal line or polygon sites, where the bisectors may also include parabolic arcs.

\section{Structural properties of order- $k$ Voronoi diagrams.}

\subsection{Edges without vertices}

Although the definition of edges and vertices resemble those of graphs embedded in $\mathcal{P}$, the situation may be quite different. The following observation is probably folklore.

Lemma 1 There exist order-k Voronoi diagrams with $k$-edges that form closed curves in $P$ and are not adjacent to any $k$-vertex.

Proof. Let us consider a prism with triangular base. Consider a line $\ell$ parallel to an edge of the prism from one base to the other, as in Fig. 1. We place $m$ sites on $\ell$, no pair of bisectors intersect and some of them define the boundary of $k$-regions. Consequently $k$-regions may have closed curve edges without vertices.

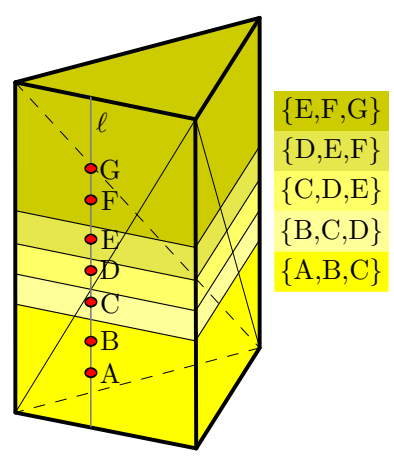

Figure 1: Example of an order-3 Voronoi diagram with all the regions without vertices.

A key property that we use below is that a $k$-edge that is not adjacent to any $k$-vertex must contain a breakpoint, as otherwise it would consist of a single arc contained in a triangle of $\mathcal{P}$, which is not possible.

\subsection{Path-connectivity of the regions}

The regions of the closest-site Voronoi diagram are path connected in any surface. The regions of the furthest-site Voronoi diagram are also path-connected in surfaces of genus zero [1]. In the Euclidean plane with point sites, any $k$-region is also path-connected, since in fact any $k$-region is 
convex. The situation is different for the general case, as shown in the following two constructions. The first construction is in the plane but requires using sites that are not points. The second construction is in a terrain, but only requires point sites.

Lemma 2 For any given $k \geq 2$, there exists a set of sites in the plane such that the order- $k$ Voronoi diagram has some disconnected regions.

Proof. In the plane, consider the segment site $s_{1}$ between the points $(-1,-7)$ and $(-1,7)$, the segment site $s_{2}$ between the points $(1,-7)$ and $(1,7)$, and the point site $s_{3}=(0,0)$. See Fig. 2 . The 2-region corresponding to the sites $s_{1}, s_{2}$ is disconnected, with a part above and a part below the line $y=0$. We can extend the construction to any $k \geq 2$ by placing $k-2$ new segment sites that are small translations of $s_{2}$.

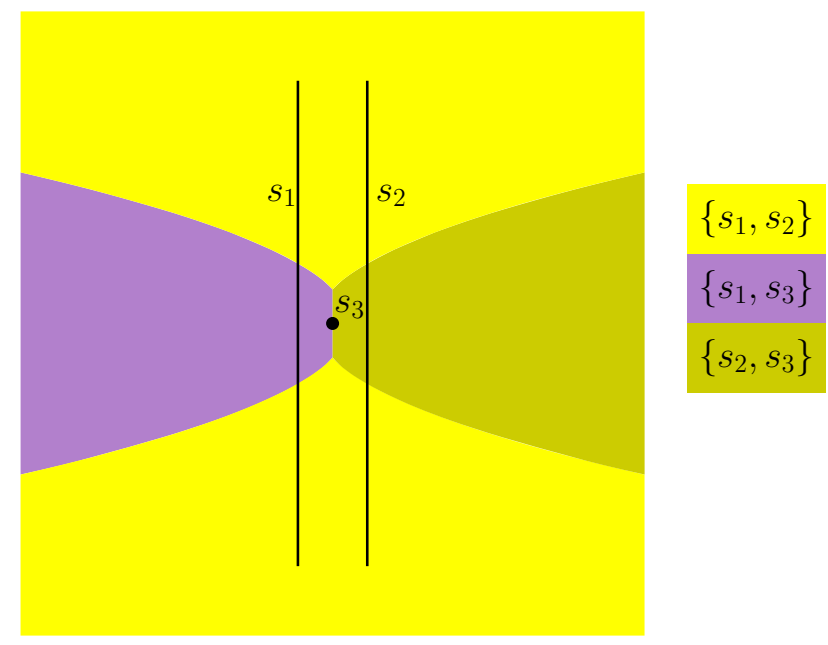

Figure 2: Example of an order-2 Voronoi diagram in the plane for segments sites with disconnected regions.

Lemma 3 For any given $k \geq 2$, there exists a polyhedral surface and a set of point sites such that the order-k Voronoi diagram has some disconnected regions.

Proof. We describe a polyhedral surface $\mathcal{P}$ embedded in 3-dimensional Euclidean space. The construction uses three squares: square $Q_{1}$ has side length 22 and corners at

$$
\begin{array}{r}
(-11,-11,0),(-11,11,0), \\
(11,11,0),(11,-11,0),
\end{array}
$$

square $Q_{2}$ has side length 20 and corners at

$$
\begin{array}{r}
(-10,-10,1000),(-10,10,1000), \\
(10,10,1000),(10,-10,1000),
\end{array}
$$

and square $Q_{3}$ has side length 18 and corners at

$$
\begin{array}{r}
(-9,-9,0),(-9,9,0), \\
(9,9,0),(9,-9,0) .
\end{array}
$$


Note that the squares are parallel to the $x y$-plane, have their centers on the line $x=y=0$, and have their sides parallel to the $x, y$ coordinate axis. Squares $Q_{1}$ and $Q_{3}$ are placed at height zero and $Q_{2}$ is at height 1000 . Let $\mathcal{P}$ be the polyhedral surface obtained by gluing the $x y$-plane minus the interior of $Q_{1}$, the pyramid with bases $Q_{1}$ and $Q_{2}$ (without the bases), the pyramid with bases $Q_{2}$ and $Q_{3}$ (without the bases), and the square $Q_{3}$. The terrain resembles a volcano. Let $\ell$ be the curve obtained by intersecting $\mathcal{P}$ with the vertical plane $y=0$. See Fig. 3 for a projection onto the $x y$-plane.

We place $3 k$ point-sites along $\ell$, as follows. First, we place $k$ sites in $Q_{3}$, symmetrically respect to its center. We then place $k$ sites along $\ell$ in each side outside $Q_{1}$, distributed in four groups: $S_{1}^{l}, S_{2}^{l}, S_{1}^{r}$ and $S_{2}^{r}$. Groups $S_{1}^{l}, S_{1}^{r}$ contain $\lfloor k / 2\rfloor$ points, while groups $S_{2}^{l}, S_{2}^{r}$ contain $\lceil k / 2\rceil$ points. Within each group, the points are regularly spaced at distance $\epsilon$, where $0<\epsilon \ll 1 / k$. The points $S_{2}^{l}$ are placed to the left of $Q_{1}$, along $\ell$, at distance $\epsilon$ from $Q_{1}$, and the points $S_{1}^{l}$ at distance $1 / 2$ from $S_{2}^{l}$. We do the same on the right of $Q_{1}$ but placing $S_{1}^{r}$ closer to $Q_{1}$ than $S_{2}^{r}$ (see Fig. 3). The construction is symmetric with respect to the plane $y=0$. (It is also symmetric with respect to the plane $x=0$ when $k$ is even.)

Consider the Voronoi region $R$ having $S_{1}^{r} \cup S_{2}^{l}$ as closest sites. First, note that $R$ is nonempty because it contains the points $(0,12,0)$ and $(0,-12,0)$ in its interior, and hence $R$ is a $k$-region. Note that no point on $\ell$ is in $R$, while the points $(0,12,0),(0,-12,0)$ are on different sides of $\ell$. This means that $R$ has at least two connected components. The construction for $k=7$ is shown in Fig. 3 ( $x y$-projection).

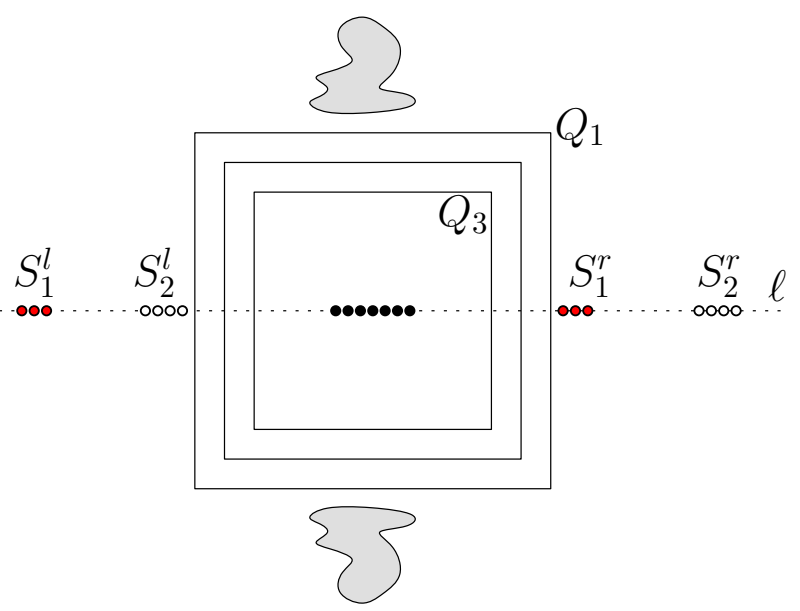

Figure 3: Example of an order-7 Voronoi diagram with disconnected regions.

\section{Complexity of order- $(\leq k)$ Voronoi diagrams}

\subsection{Upper bounds}

In this section we will give bounds for the complexity of $V_{\leq k}(S)$. Our approach is similar to the approach used by Clarkson and Shor [5] or Sharir [13]. Let $B_{j}$ be the total number of $j$-breakpoints, and let $B_{\leq k}$ be the number of $j$-breakpoints for $j=1, \ldots, k$. Similarly, let $V_{j}$ be the number of $j$-vertices and let $V_{\leq k}$ be the total number of $j$-vertices for $j=1, \ldots, k$. 
Lemma 4 The complexity of the order- $k$ Voronoi diagram is $O\left(B_{k}+V_{k}\right)$.

Proof. Let $E_{k}$ and $F_{k}$ be the number of $k$-edges and $k$-faces, respectively.

Each $k$-edge that is not adjacent to a $k$-vertex (cf. Lemma 1) contains some $k$-breakpoints. Therefore, the number of $k$-edges that are not adjacent to any $k$-vertex is bounded by $B_{k}$. The number of $k$-edges adjacent to some $k$-vertex is bounded by $3 V_{k}$ because each $k$-vertex is adjacent to at most three $k$-edges. Therefore, we conclude that $E_{k}=O\left(B_{k}+V_{k}\right)$.

To bound $F_{k}$, we can use Euler's formula to obtain the bound $F_{k} \leq E_{k}-V_{k}+2-g$, where $g \geq 0$ is the genus of the surface ${ }^{2}$. Therefore $F_{k} \leq E_{k}+2=O\left(E_{k}\right)$. We conclude that the complexity of the order- $k$ Voronoi diagram, defined as $F_{k}+E_{k}+V_{k}+B_{k}$, is $O\left(B_{k}+V_{k}\right)$.

Lemma 5 We have $B_{\leq k}=O(k n(k n+m))$ for any $1 \leq k \leq m-1$.

Proof. Draw a random sample $R$ of $S$, by independently drawing each element of $S$ with probability $p$. We will set below $p$ to an appropriate value. Let $B(R)$ be the number of breakpoints of the order1 Voronoi diagram of $R$ in $\mathcal{P}$. From the bounds on the complexity of order-1 Voronoi diagrams, discussed in Section 1, we know that $B(R)=O\left(n^{2}+|R| n\right)$. Since $|R|$ follows a binomial distribution, we have

$$
\mathbb{E}[|R|]=m p,
$$

and therefore

$$
\mathbb{E}[B(R)]=O\left(n^{2}+n m p\right) .
$$

Consider a breakpoint $b$ in some $j$-edge of the order- $j$ Voronoi diagram of $S$. Let $s, s^{\prime}$ be the two sites defining the bisector that contains $b$. Consider the set $S_{b}$ of $j-1$ sites distinct from $s, s^{\prime}$ that are at distance at most $d(b, s)$ from $b$. The point $b$ is a breakpoint in the order-1 Voronoi diagram of the random sample $R$ if and only if $s, s^{\prime}$ are in $R$ and none of the $j-1$ sites $S_{b}$ is in $R$. Therefore, the probability that $b$ is a breakpoint in the order-1 Voronoi diagram of $R$ is precisely $p^{2}(1-p)^{j-1}$. By linearity of expectation we then have

$$
\begin{aligned}
\mathbb{E}[B(R)] & =\sum_{j=1}^{m-1} B_{j} p^{2}(1-p)^{j-1} \\
& \geq p^{2} \sum_{j=1}^{k} B_{j}(1-p)^{j-1} \\
& \geq p^{2}(1-p)^{k-1} \sum_{j=1}^{k} B_{j} \\
& =p^{2}(1-p)^{k-1} B_{\leq k} .
\end{aligned}
$$

Rearranging and substituting equation (1) we see

$$
\begin{aligned}
B_{\leq k} & \leq \frac{\mathbb{E}[B(R)]}{p^{2}(1-p)^{k-1}} \\
& =\frac{O\left(n^{2}+n m p\right)}{p^{2}(1-p)^{k-1}} .
\end{aligned}
$$

\footnotetext{
${ }^{2}$ There is equality only for nonorientable surfaces without boundary where each $k$-face is a topological disk. For orientable surfaces we can replace the term $g$ by $2 g$.
} 
Finally, setting $p=1 / k$ we obtain

$$
\begin{aligned}
B_{\leq k} & =O\left(\frac{n^{2}+n m / k}{(1 / k)^{2}(1-1 / k)^{k-1}}\right) \\
& =O\left(k^{2} n^{2}+k n m\right),
\end{aligned}
$$

where we have used that

$$
\frac{1}{(1-1 / k)^{k-1}}=\left(\frac{k}{k-1}\right)^{k-1} \leq e
$$

Lemma 6 We have $V_{\leq k}=O\left(k^{2} m\right)$ for any $1 \leq k \leq m-1$.

Proof. This proof is very similar to the previous one. Draw a random sample $R$ from $S$, by independently drawing each element of $S$ with probability $p$. Let $V(R)$ be the number of vertices of the order-1 Voronoi diagram of $R$ in $\mathcal{P}$. From the known bounds, as discussed in Section 1, we have $V(R)=O(|R|)=O(m p)$.

Consider a $j$-vertex $v$, and let $s_{1}, s_{2}, s_{3}$ be the three sites defining $v: v$ is in the intersection of the bisectors $\beta\left(s_{1}, s_{2}\right), \beta\left(s_{1}, s_{3}\right), \beta\left(s_{2}, s_{3}\right)$. Consider the set $S_{v}$ of sites distinct from $s_{1}, s_{2}, s_{3}$ that are at distance at most $d\left(v, s_{1}\right)$ from $v$. The cardinality of $S_{v}$ is either $j-2$ (when $v$ is also a $(j-1)$-vertex) or $j-1$ (when $v$ is also a $(j+1)$-vertex). The point $v$ is a vertex in the order- 1 Voronoi diagram of the random sample $R$ if and only if $s_{1}, s_{2}, s_{3}$ are in $R$ and none of the sites $S_{v}$ are in $R$. Therefore, the probability that $v$ is a vertex in the order-1 Voronoi diagram of $R$ is precisely $p^{3}(1-p)^{\left|S_{v}\right|} \geq p^{3}(1-p)^{j-1}$. Since a $j$-vertex is also a $(j-1)$-vertex or a $(j+1)$-vertex, each vertex is counted twice in the sum $V_{1}+V_{2}+\cdots+V_{m-1}$. By linearity of expectation we then have

$$
\begin{aligned}
\mathbb{E}[V(R)] & \geq \frac{1}{2} \sum_{j=1}^{m-1} V_{j} p^{3}(1-p)^{j-1} \\
& \geq \frac{1}{2} p^{3} \sum_{j=1}^{k} V_{j}(1-p)^{j-1} \\
& =\frac{1}{2} p^{3}(1-p)^{k-1} V_{\leq k} .
\end{aligned}
$$

As in the previous proof, we rearrange, substitute $V(R)=O(m p)$, and set $p=1 / k$ to get

$$
\begin{aligned}
V_{\leq k} & \leq \frac{2 \cdot \mathbb{E}[V(R)]}{p^{3}(1-p)^{k-1}} \\
& =\frac{2 m / k}{(1 / k)^{3}(1-1 / k)^{k-1}} \\
& =O\left(k^{2} m\right) .
\end{aligned}
$$

Combining Lemmas 4-6 we can conclude our main result:

Theorem 1 Let $\mathcal{P}$ be a polyhedral surface with $n$ triangles, let $S$ be a set of $m$ sites on $\mathcal{P}$, and let $k$ be an integer between 1 and $m-1$. The complexity of $\operatorname{Vor}_{\leq k}(S)$ is $O\left(k^{2} n^{2}+k^{2} m+k n m\right)$. 


\subsection{Lower bounds}

We will show that the bound of Theorem 1 is asymptotically optimal in the worst case. For this, we give three constructions, each showing the need of one summand in the bound $O\left(k^{2} n^{2}+k^{2} m+k n m\right)$. Our constructions only use terrains, and hence the upper bounds in Theorem 1 are tight even for terrains.

We describe a construction attaining complexity $\Omega\left(m k^{2}\right)$. Consider the Euclidean plane and observe that the complexity of $\operatorname{Vor}_{\leq k}(S)$ for a set $S$ of $m$ points is $\Omega\left(m k^{2}\right)$ [7]. Clipping the plane with a large enough rectangle, so that $n=O(1)$, we obtain the desired bound.

We describe a construction attaining complexity $\Omega(k n m)$. Consider the Euclidean plane, and take the point sites $s_{i}=(i, 0), i=1, \ldots, m$. Sites with consecutive indices, like $s_{i}, s_{i+1}, \ldots, s_{i+j-1}$, define a $j$-region, which is a vertical strip. Therefore, $V o r_{j}$ consists of $m-j+1$ regions, and $V o r_{\leq k}$ has $\Omega(\mathrm{km})$ regions. Clip the plane with a sufficiently large axis-parallel rectangle, and triangulate it with $n$ 'long' edges that connect the left boundary to the right boundary. Each such 'long' edge intersects each region in $V o r_{\leq k}$, and hence the complexity of $\operatorname{Vor}_{\leq k}$ is $\Omega(k m n)$.

We describe a construction attaining complexity $\Omega\left(k^{2} n^{2}\right)$. Let us assume for simplicity that $m$ is even. Consider the Euclidean plane and define the $m / 2$ point sites $s_{i}=(2+\varepsilon \cdot i, 0)$, for $i=1, \ldots, m / 2$, where $\varepsilon>0$ is a sufficiently small value to be set below. Define also the sites $s_{i}^{\prime}$ by $s_{i}^{\prime}=-s_{i}$, for $i=1, \ldots, m / 2$. Note that the bisector between $s_{i}$ and $s_{i}^{\prime}$ is the line $x=0$. Let us place $n$ small rectangular obstacles along the line $x=1 / 2$. For concreteness, let $R_{t}$ be the axis-aligned square with center $(1 / 2, t)$ and side length $1 / n$. We erect an almost vertical wall over each of the edges of each $R_{t}$. This is like effectively considering each $R_{t}$ as an obstacle. Note that there is a line through $s_{i}$ that separates the rectangle $R_{t}$ from $R_{t+1}$, for any $i=1, \ldots, m / 2$ and $t=1, \ldots, n$. See Fig. 4.

Let $\beta$ denote the bisector between sites $s_{1}$ and $s_{1}^{\prime}$. The bisector $\beta$ is not a line anymore, but contains $\Theta(n)$ straight-line arcs - contained in the line $x=0$-and $\Theta(n)$ hyperbolic arcs - contained in the halfplane $x \geq 0$. Consider a value $\delta>0$ sufficiently small such that the vertical line $x=\delta$ intersects each hyperbolic arc in $\beta$. Let $\ell$ denote the vertical line $x=\delta / 2$. We can take $\varepsilon$ to be a sufficiently small value such that the line $\ell$ intersects all the hyperbolic arcs in any of the bisectors between a site $s_{i}$ and a site $s_{j}^{\prime}$, for any $i, j \in\{1, \ldots, m / 2\}$. This is possible because, looking at a bounded portion of the plane, the bisector $\beta\left(s_{1}, s_{1}^{\prime}\right)$ changes continuously with a small perturbation of the sites $s_{1}, s_{1}^{\prime}$. Therefore, the line $\ell$ intersects $\Theta(n)$ times the bisector $\beta\left(s_{i}, s_{j}^{\prime}\right)$, for any $i, j \in\{1, \ldots, m / 2\}$. We clip the surface with a large enough rectangle, and triangulate it adding $n$ edges that are parallel to $\ell$ and very close together; each of the added edges should intersect $\Theta(n)$ times any bisector $\beta\left(s_{i}, s_{j}^{\prime}\right)$.

Let $S_{i, j}$ denote the subset of sites

$$
\left\{s_{1}, s_{2}, \ldots, s_{i}\right\} \cup\left\{s_{1}^{\prime}, s_{2}^{\prime}, \ldots, s_{j}^{\prime}\right\} .
$$

Note that a point $p$ in the plane whose $x$ coordinate is between -1 and 1 is closer to $s_{i}$ than to $s_{i+1}$ for any $i \in\{1, \ldots, m / 2-1\}$. Similarly, such point is closer to $s_{j}^{\prime}$ than to $s_{j+1}^{\prime}$. This implies that the $i+j$ sites $S_{i, j}$ define a nonempty $(i+j)$-region in $\operatorname{Vor}_{i+j}(S)$ whose boundary is determined by the bisectors $\beta\left(s_{i}, s_{j+1}^{\prime}\right)$ and $\beta\left(s_{i+1}, s_{j}^{\prime}\right)$. Therefore, the boundary of $V\left(S_{i, j}\right)$ has complexity $\Omega\left(n^{2}\right)$ because bisector $\beta\left(s_{i}, s_{j+1}^{\prime}\right)$ is intersected $n$ times by each of the $n$ edges parallel to $\ell$. We conclude that any set $S_{i, j}$ defines a $(i+j)$-region of complexity $\Omega\left(n^{2}\right)$. Since $k \leq m$, there are $\Theta\left(k^{2}\right)$ pairs of values $i, j \in\{1, \ldots, m / 2\}$ adding to at most $k$. This means that $\operatorname{Vor}_{\leq k}$ has at least $\Omega\left(k^{2}\right)$ regions, each of complexity $\Omega\left(n^{2}\right)$. Therefore in this construction the complexity of $\operatorname{Vor}_{\leq k}$ is $\Omega\left(k^{2} n^{2}\right)$. 


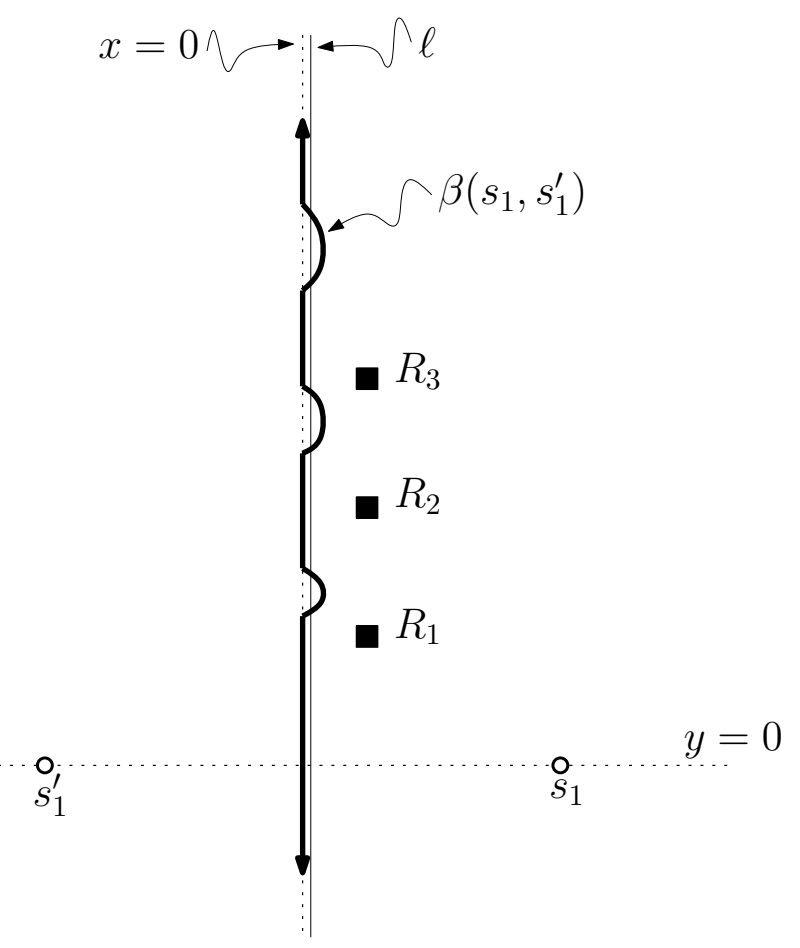

Figure 4: The lower bound for $\Omega\left(k^{2} n^{2}\right)$ when $n=3$; the hyperbolic arcs are symbolic and only the sites $s_{1}, s_{1}^{\prime}$ are shown.

\section{Complexity of order- $k$ Voronoi diagrams on realistic terrains}

We next restrict ourselves to so-called realistic terrains [9]. Let $\alpha, \beta, \gamma, \delta$ be constants independent of $n, m$. A realistic terrain is a terrain where:

1. the domain is a rectangle whose aspect ratio is upper bounded by $\alpha$;

2. the angle of any segment contained in $\mathcal{T}$ with the horizontal plane is upper bounded by $\beta<\frac{\pi}{2}$;

3. the ratio between the longest and the shortest edge in the $x y$-projection of $\mathcal{T}$ is upper bounded by $\gamma$;

4. the triangulation of the domain induced by the $x y$-projection is a $\gamma$-low-density triangulation: any square $Q$ intersects at most $\gamma$ edges of length greater than the side length of $Q$.

Aronov et al. [2] have recently showed that in a realistic terrain the closest-site Voronoi diagram of $m$ point sites has complexity $O(n+m \sqrt{n})$; this improves the previous bound by Moet et al. [9]. Using this bound in Lemma 5 we obtain the following result.

Lemma 7 In a realistic terrain $B_{\leq k}=O\left(k^{2} n+k \sqrt{n} m\right)$ for any $1 \leq k \leq m-1$.

Proof. Consider the proof of Lemma 5 for a realistic terrain. Using the bound

$$
B(R)=O(n+|R| \sqrt{n})=O(n+\sqrt{n} m p),
$$


instead of the equation (1), one readily obtains

$$
B_{\leq k}=O\left(k^{2} n+k \sqrt{n} m\right),
$$

Combining Lemmas 4, 6, and 7 we can conclude the following.

Theorem 2 Let $\mathcal{T}$ be a realistic terrain with $n$ triangles, let $S$ be a set of $m$ point sites, and let $k$ be an integer between 1 and $m-1$. The complexity of $\operatorname{Vor}_{\leq k}(S)$ is $O\left(k^{2} n+k \sqrt{n} m+k^{2} m\right)$.

\section{Conclusions}

We have have given tight bounds for the combinatorial complexity of order- $(\leq k)$ Voronoi diagrams in triangulated surfaces. Our upper bound of course also holds for the combinatorial complexity of the order- $k$ Voronoi diagram. Obtaining better upper bounds for the order- $k$ Voronoi diagram seems a much more challenging problem, even if restricted only to terrains or realistic terrains.

\section{Acknowledgements}

We are grateful to the anonymous referees for their corrections and the construction in Lemma 2 .

\section{References}

[1] B. Aronov, M. van Kreveld, R. W. van Oostrum, and K. Varadarajan. Facility location on terrains. Discrete and Computational Geometry, 30:257-372, 2003.

[2] B. Aronov, M. de Berg, and S. Thite. The complexity of bisectors and Voronoi diagrams on realistic terrains. Algorithms - ESA 2008, 16th Annual European Symposium on Algorithms, LNCS 5193:100-111, 2008.

[3] F. Aurenhammer. Voronoi diagrams: a survey of a fundamental geometric data structure. ACM Comput. Surv., 23:345-405, 1991.

[4] F. Aurenhammer and R. Klein. Voronoi diagrams. In: J. R. Sack, J. Urrutia (Eds.), Handbook of Computational Geometry, 2000, Elsevier, 201-290.

[5] K. L. Clarkson and P. W. Shor. Application of random sampling in computational geometry, II, Discrete and Computational Geometry, 4:387-421, 1989.

[6] M. Fort and J. A. Sellarès. Generalized higher-order Voronoi diagrams on polyhedral terrains. 4th International Symposium on Voronoi Diagrams in Science an Engineering, pages 74-84, 2007.

[7] D. T. Lee. On $k$-nearest neighbor Voronoi diagrams in the plane. IEEE Trans. Computers, 31:478-787, 1982.

[8] J. S. B. Mitchell, D. M. Mount, and C. H. Paparimitriou. The discrete geodesic problem. SIAM J. Computation, 16:647-668, 1987. 
[9] E. Moet, M. van Kreveld, and F. van der Stappen, On realistic terrains. In SCG '06: Proceedings of the 22nd Symposium on Computational Geometry, pages 177-186, 2006.

[10] D. M. Mount. Voronoi diagrams on the surface of a polyhedron. Technical report, N.1496, University of Maryland, 1985.

[11] A. Okabe, B. Boots, K. Sugihara, and S. N. Chiu. Spatial Tessellation: Concepts and Application of Voronoi Diagrams. John Wiley and Sons, 2000.

[12] D. Schmitt and J. C. Spehner. Order- $k$ Voronoi diagrams, $k$-sections, and $k$-sets. In $J C D C G$ '98: Japanese Conference on Discrete and Computational Geometry, LNCS 1763, pages 290304, 1998.

[13] M. Sharir. The Clarkson-Shor technique revisited and extended. Combinatorics, Probability E Computing, 12:191-201, 2003.

[14] V. Surazhsky, T. Surazhsky, D. Kirsanov, S. J. Gortler, and H. Hoppe. Fast exact and approximate geodesics on meshes. ACM Trans. Graph., 24(3):553-560, 2005. 